\title{
Risk factors for and outcomes of prolonged mechanical ventilation in patients received DeBakey type I aortic dissection repairment
}

\author{
Min Ge", Zhigang Wang", Tao Chen, Yongqing Cheng, Jiaxin Ye, Lichong Lu, Cheng Chen, \\ Dongjin Wang
}

Department of Cardio-thoracic Surgery, Nanjing Drum Tower Hospital, The Affiliated Hospital of Nanjing University Medical School, Nanjing, China

Contributions: (I) Conception and design: D Wang; (II) Administrative support: D Wang, M Ge; (III) Provision of study materials or patients: M Ge, T Chen, Y Cheng, C Chen; (IV) Collection and assembly of data: Z Wang, J Ye, L Lu; (V) Data analysis and interpretation: Z Wang, M Ge; (VI) Manuscript writing: All authors; (VII) Final approval of manuscript: All authors.

\#These authors contributed equally to this work.

Correspondence to: Dongjin Wang. Department of Cardio-thoracic Surgery, Nanjing Drum Tower Hospital, The Affiliated Hospital of Nanjing University Medical School, Nanjing 210008, China. Email: glyywdj@163.com.

\begin{abstract}
Background: This study aimed to identify risk factors for prolonged mechanical ventilation (PMV) and its association with disease prognosis following acute DeBakey type I aortic dissection surgery.

Methods: A total of 582 patients who received emergency surgery for acute DeBakey type I aortic dissection from 2014 to 2018 were enrolled in this study. Mechanical ventilation period after surgery longer than 48 hours was defined as postoperative PMV. Multiple logistic regression analysis was used to identify risk factors for PMV. This study also compared short- and long-term outcomes in patients who developed PMV with patients who did not develop this complication. To identify and compare long-term cumulative survival rate, Kaplan-Meier survival curve was plotted.

Results: Among all enrolled patients, 259 (44.5\%) received PMV treatment. Our data suggested that the length of intensive care unit and hospital stay were longer for patients who received PMV treatment. Thirtyday mortality was also higher in patients with PMV than in patients without PMV. Elevated leukocyte count and increased serum cystatin $\mathrm{C}$ level upon admission, lower preoperative platelet count and longer cardiopulmonary bypass (CPB) duration were identified as risk factors for PMV. Interestingly, our data suggested that there was no significant difference of survival rate between patients with or without PMV history.

Conclusions: PMV after DeBakey type I aortic dissection repair surgery was a common complication and associated with increased short-term mortality rate but did not affect long-term mortality rate. Elevated preoperative leukocyte count, increased preoperative serum cystatin C level, lower preoperative platelet count and longer CPB duration were risk factors for PMV.
\end{abstract}

Keywords: Prolonged mechanical ventilation (PMV); aortic dissection; outcomes

Submitted Aug 24, 2020. Accepted for publication Dec 03, 2020.

doi: $10.21037 /$ jtd-20-2736

View this article at: http://dx.doi.org/10.21037/jtd-20-2736

\section{Introduction}

Acute DeBakey type I aortic dissection is a cardiovascular catastrophic event with high mortality that requires emergency repair surgery. Compared to other cardiovascular disease, patients with DeBakey type
I aortic dissection are more likely to experience perioperative hemodynamic instability, transfusion and other postoperative complications such as respiratory dysfunction (1). The respiratory dysfunction can be severe and often requires prolonged mechanical ventilation 
(PMV). Patients who received PMV were associated with longer intensive care unit (ICU) stay and worse prognosis (2-4). Identification of PMV risk factors may allow for optimization of perioperative management strategies and eventually improve clinical outcomes.

Several studies have been conducted to identify risk factors for and prognosis of PMV following other types of cardiac surgery (2). In cardiac revascularization and valve surgery, variables such as older age, chronic obstructive pulmonary disease, emergency surgery, and longer cardiopulmonary bypass $(\mathrm{CPB})$ time were identified as predictors for PMV. PMV was also proved to associate with increased hospital stay, mortality and, consequently, costs in this study (5). While for type A aortic dissection (TAAD) aortic repair surgery, lower preoperative platelet count and a higher serum lactate level were risk factors for PMV (4). Postoperative longer intubation duration was also associated with poor long-term survival in adult patients undergoing surgery for TAAD (6). However, little is known about patients who received PMV treatment after acute DeBakey type I aortic dissection surgery. This retrospective study aimed to identify risk factors for PMV following acute DeBakey type I aortic dissection surgery and to assess its influence on both short- and long-term outcomes. We present the following article in accordance with the STROBE reporting checklist (available at http://dx.doi. org/10.21037/jtd-20-2736).

\section{Methods}

\section{Patients}

The Ethics Committee of Nanjing University approved this study (No. BL2014004) and waived the need for individual informed consent considering the retrospective nature of the study. The study was conducted in accordance with the Declaration of Helsinki (as revised in 2013). Aortic dissection was defined as acute if chest pain or other related symptoms were presented less than 14 days before the operation. A total of 597 patients with acute DeBakey type I aortic dissection underwent emergency surgery at the authors' hospital between January 2014 and December 2018 were retrospectively screened for the study. Surgery was performed after a mean of $17.0 \mathrm{~h}$ after symptoms onset. The diagnosis of DeBakey type I aortic dissection was confirmed with computed tomographic angiography. Ten patients who died within $24 \mathrm{~h}$ after surgery and 5 patients who did not have complete postoperative data were excluded from the study. A total of 582 patients (433 men, 149 women) with a median age of $52.0(43.8,61.0)$ years were eventually included in this study. PMV was defined as total intubation and ventilation time for at least 48 hours at any time during the postoperative stay. Preoperative routine blood examinations such as leukocyte count and platelet count were recorded based on the results of the initial laboratory test after admission before surgery.

\section{Surgical procedures}

The surgical procedures has been described in detail previously $(7,8)$. In brief, all procedures were carried out with median sternotomy and total $\mathrm{CPB}$. The right axillary artery was dissected for $\mathrm{CPB}$ and selective cerebral perfusion. During the cooling phase, the ascending aorta was clamped. Cold blood cardioplegia was infused by both anterograde and retrograde infusion method. Arch repair method including hemiarch replacement, island arch replacement, total arch replacement, triple-branched stent and fenestrated stent were selected as appropriate.

\section{Respiratory weaning protocol}

All patients were transferred to ICU and received mechanical ventilation (pressure-control mode) at an appropriate inspired oxygen fraction to maintain arterial oxygen saturation $>95 \%$ after surgery. The extubation criteria were determined as follow: full weakness, stable hemodynamics without excessive bleeding, normal acidbase balance, complete recovery from muscle relaxation, tidal volume $>6 \mathrm{~mL} / \mathrm{kg}$, respiratory rate $<30 / \mathrm{min}$, arterial oxygen tension $>200 \mathrm{mmHg}$, and arterial carbon dioxide tension $<50 \mathrm{mmHg}$ (4). If patients experienced hypoxemia after extubation, non-invasive positive-pressure ventilation, or high flux inhalation of oxygen was applied.

\section{Statistical analysis}

Data analysis was performed using SPSS software (Version 25.0, IBM Corp., Armonk, NY, USA). All continuous variables were expressed as mean \pm standard deviation or median (interquartile range) based on whether the variables were normally distributed and compared with Student $t$-test (normal distribution) or Mann-Whitney $U$-test (non-normal distributions). Numbers (percentage) were compared with Pearson chi-square or Fisher exact test. Risk factors for PMV were identified using binary logistic regression model. 
Table 1 Comparison of preoperative variables

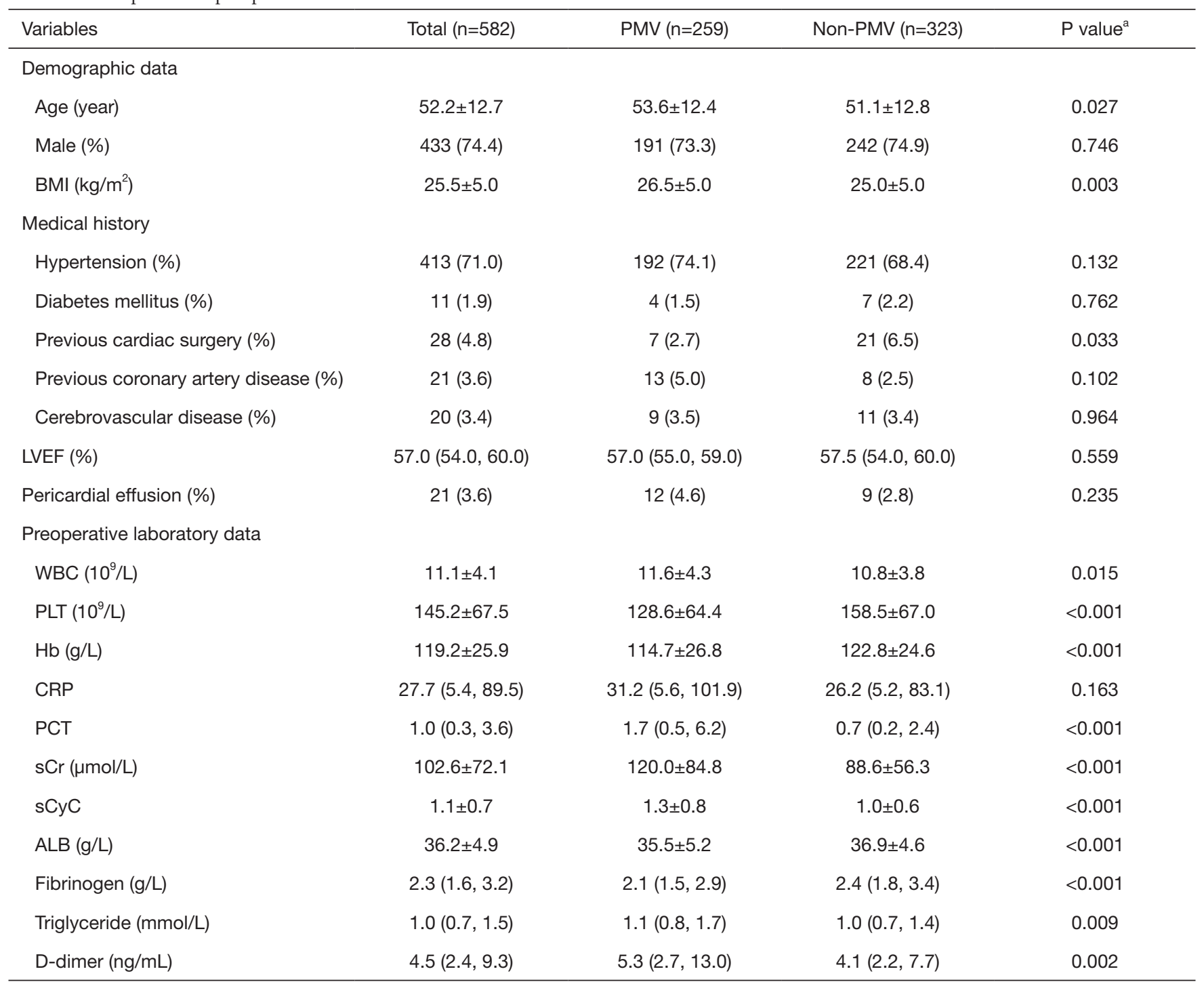

Data presented as $\mathrm{n}(\%)$; median (IQR). ${ }^{a}$, $\mathrm{P}$ values indicate differences between PMV and Non-PMV groups. $\mathrm{P}<0.05$ was considered statistically significant. PMV, prolonged mechanical ventilation; BMI, body mass index; LVEF, left ventricular ejection fraction; WBC, white blood cell; PLT, platelet; $\mathrm{Hb}$, hemoglobin; CRP, c reactive protein; $\mathrm{s} C r$, serum creatinine; sCyC, serum cystatin C; ALB, albumin;

Variables associated with PMV that had a $\mathrm{P}$ value $<0.20$ in the univariable analysis were further examined using binary logistic regression (stepwise enter method). Survival rate was estimated with Kaplan-Meier plot, and intergroup comparisons were examined by log-rank test. $\mathrm{P}$ value $<0.05$ was considered to be statistically significant.

\section{Results}

The preoperative characteristics of all 582 patients were summarized in Table 1. Among all enrolled patients, 259
(44.5\%) required PMV. Our data suggested that the following preoperative variables were significantly different between patients with PMV and without PMV: years of age $(\mathrm{P}=0.027)$, $\mathrm{BMI}(\mathrm{P}=0.003)$, leukocyte count $(\mathrm{P}=0.015)$, hemoglobin $(\mathrm{P}<0.001)$, platelet count $(\mathrm{P}<0.001)$, serum creatinine $(\mathrm{P}<0.001)$, serum cystatin $\mathrm{C}(\mathrm{s} C y \mathrm{C})(\mathrm{P}<0.001)$, albumin $(\mathrm{P}<0.001)$, fibrinogen $(\mathrm{P}<0.001)$, triglyceride $(\mathrm{P}=0.009)$ and $\mathrm{D}$-dimer $(\mathrm{P}=0.002)$.

As presented in Table 2, intraoperative features like CPB time $(\mathrm{P}<0.001)$, aortic cross-clamp time $(\mathrm{P}=0.011)$, and deep hypothermic circulatory arrest (DHCA) time $(\mathrm{P}=0.004)$ 
Table 2 Comparison of operative variables

\begin{tabular}{lcccc}
\hline Variables & Total $(\mathrm{n}=582)$ & PMV $(\mathrm{n}=259)$ & Non-PMV $(\mathrm{n}=323)$ & $\mathrm{P}$ value ${ }^{\mathrm{a}}$ \\
\hline TAR $(\%)$ & $368(63.2)$ & $165(63.7)$ & $203(62.8)$ & 0.831 \\
CABG/MVR/MVP/TVP (\%) & $53(9.1)$ & $30(11.6)$ & $63(7.1)$ & 0.063 \\
Aortic valve (\%) & $155(26.6)$ & $259.3)$ & $92(28.5)$ & 0.259 \\
CPB time (min) & $245.2 \pm 69.3$ & $177.4 \pm 62.8$ & $165.4 \pm 56.3$ & $<0.001$ \\
Aortic cross-clamp time (min) & $170.7 \pm 59.5$ & $31.3 \pm 11.7$ & $28.8 \pm 12.5$ & 0.011 \\
DHCA time (min) & $29.9 \pm 12.2$ & 0.004 & \\
\hline
\end{tabular}

Data presented as $\mathrm{n}(\%)$; median (IQR). ${ }^{a}, \mathrm{P}$ values indicate differences between $\mathrm{PMV}$ and Non-PMV groups. $\mathrm{P}<0.05$ was considered statistically significant. TAR, total arch replacement; CABG, coronary artery bypass graft; MVR, mitral valve replacement; MVP, mitral valvuloplasty; TVP, tricuspid valvuloplasty; CPB, cardiopulmonary bypass; DHCA, deep hypothermic circulatory arrest.

Table 3 Comparison of postoperative variables

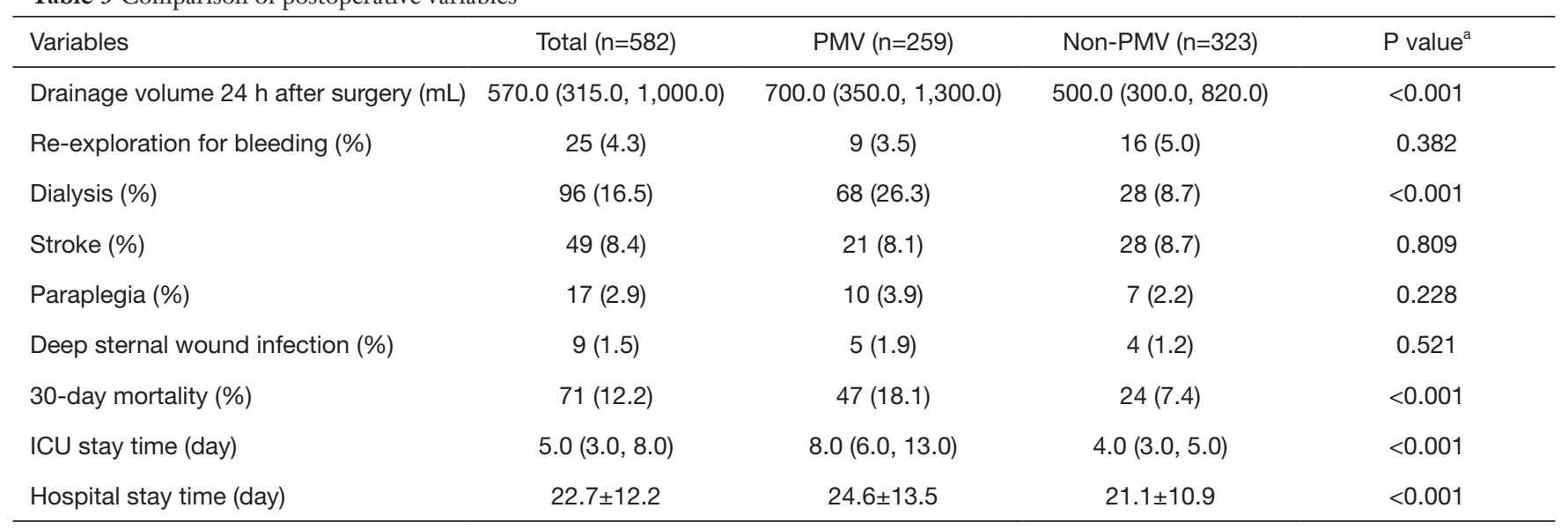

Data presented as $\mathrm{n}(\%)$; median (IQR). ${ }^{a}$, $\mathrm{P}$ values indicate differences between PMV and Non-PMV groups. P<0.05 was considered statistically significant. PMV, prolonged mechanical ventilation; ICU, intensive care unit.

were significantly prolonged in patients with PMV.

The postoperative features and morbidities were shown in Table 3. We discovered that patients with PMV had more drainage volume $24 \mathrm{~h}$ after surgery $(\mathrm{P}<0.001)$, higher dialysis rate $(\mathrm{P}<0.001)$, increased 30 -day mortality $(\mathrm{P}<0.001)$, longer ICU $(\mathrm{P}<0.001)$ and hospital stays $(\mathrm{P}<0.001)$.

Multivariable analysis indicated that elevated preoperative leukocyte count (OR 1.103; 95\% CI: $1.008-$ 1.206; $\mathrm{P}=0.033$ ), higher level of preoperative serum cystatin $\mathrm{C}(\mathrm{sCyC})$ [odds ratio (OR) 2.337; 95\% confidence interval (CI): 1.249-4.373; $\mathrm{P}=0.008]$, lower preoperative platelet count (OR 0.990; 95\% CI: 0.982-0.998; $\mathrm{P}=0.013$ ) and longer CPB duration (OR 1.011; 95\% CI: 1.001-1.022; $\mathrm{P}=0.028$ ) were independent predictors for PMV (Table 4).

By December 2019, 508 of 582 (87.3\%) patients had been followed up with a median of 29 months. Thirtyeight patients were lost from the cohort and excluded from the survival analysis. Thirty-two patients died during the follow-up period with 16 in each group. Kaplan-Meier survival analysis suggested that there was no significant difference of cumulative survival rate between patients with or without $\mathrm{PMV}(\mathrm{P}=0.728)$ (Figure 1).

\section{Discussion}

PMV after cardiac surgery is a common yet frightening complication (6) that might result from respiratory failure, neurologic insult, or systemic sepsis. To investigate the risk factors for and outcomes of PMV after DeBakey type I aortic dissection repair surgery, we retrospectively reviewed a total of 582 patients and discovered that this complication 
Table 4 Binary logistic regression analysis on factors for the groups with and without PMV

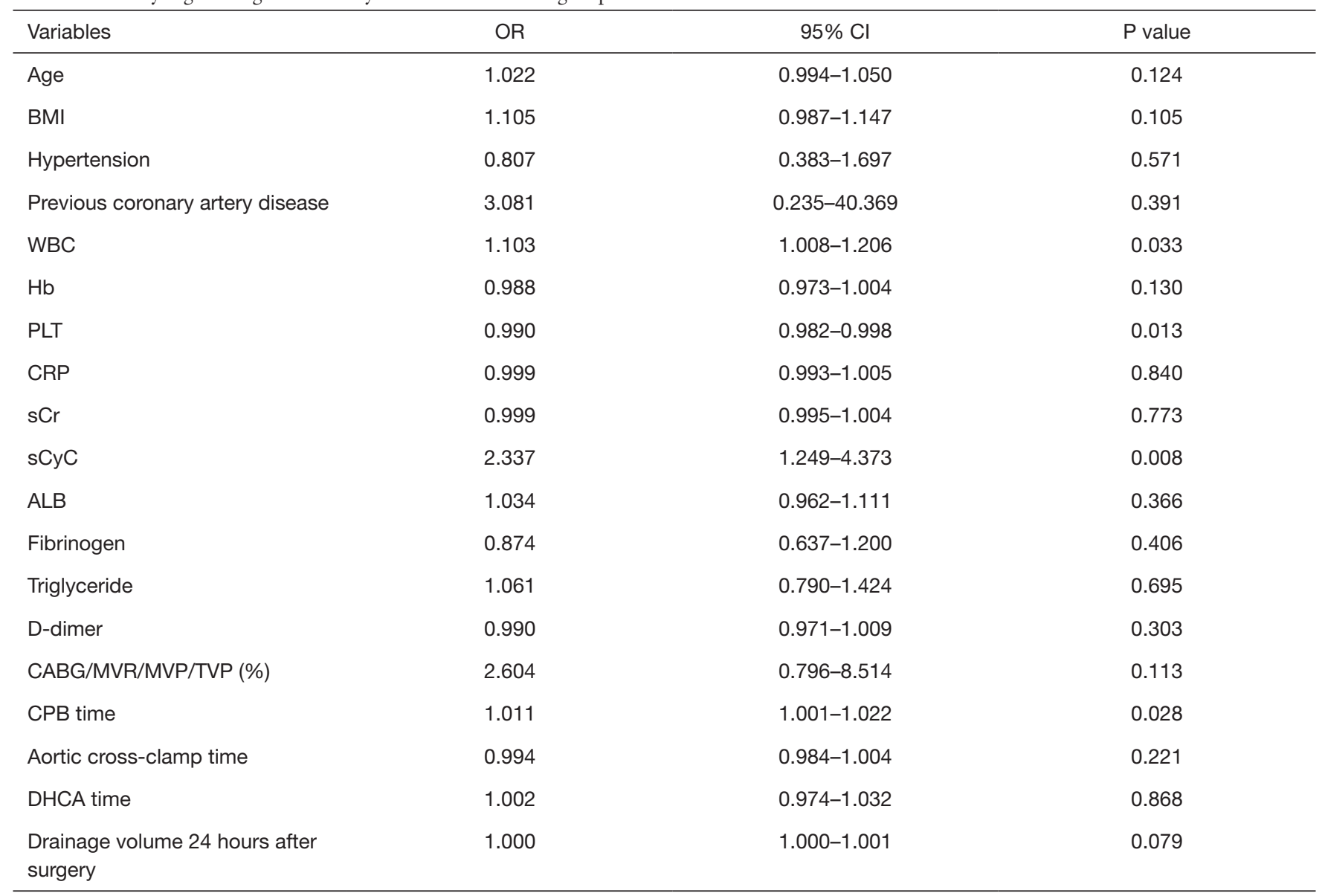

$\mathrm{P}<0.05$ was considered statistically significant. $\mathrm{PMV}$, prolonged mechanical ventilation; $\mathrm{BMI}$, body mass index; WBC, white blood cell; $\mathrm{Hb}$, hemoglobin; PLT, platelet; CRP, c reactive protein; sCr, serum creatinine; sCyC, serum cystatin C; ALB, albumin; CABG, coronary artery bypass graft; MVR, mitral valve replacement; MVP, mitral valvuloplasty; TVP, tricuspid valvuloplasty; CPB, cardiopulmonary bypass; DHCA, deep hypothermic circulatory arrest.; OR, odds ratio; $\mathrm{Cl}$, confidence interval.

was associated with increased short-term mortality rate but did not affect long-term mortality rate. Furthermore, our data suggested that elevated preoperative leukocyte count, increased preoperative serum cystatin C level, lower preoperative platelet count and longer CPB duration were identified as risk factors for PMV.

In consistent with previous studies, an increased preoperative leukocyte count was identified as a risk factor for PMV (3). The exact mechanism of how leukocyte contribute to PMV remains to be fully determined. However, the processes may include unique capillary structure of the lung, neutrophil deformability, and adhesion molecules (9). The pulmonary vascular bed stores $50-60 \%$ of total circulating neutrophils, a disturbance of local neutrophil equilibrium might directly induce tissue damage (10). As a matter of fact, $\mathrm{Xu}$ et al. reported that using high-dose ulinastatin (neutrophil inhibitor) reduced pulmonary injury and improved the pulmonary function after CPB under DHCA (11). Similarly, Alexiou et al. showed that the use of a leukocyte filter throughout $\mathrm{CPB}$ protected the pulmonary function (12). It is possible that appropriate neutrophil inhibitor or filtration that decreases leukocyte number might help decreasing the risk for PMV.

A lower preoperative platelet count was identified as another risk factor for PMV. This finding was consistent with previous studies (4). A possible explanation for this finding is that platelet plays a crucial role in the pathogenesis of acute lung injury (13). Platelet activation in acute aortic dissection might further affect attached leukocytes and endothelial cells by releasing 


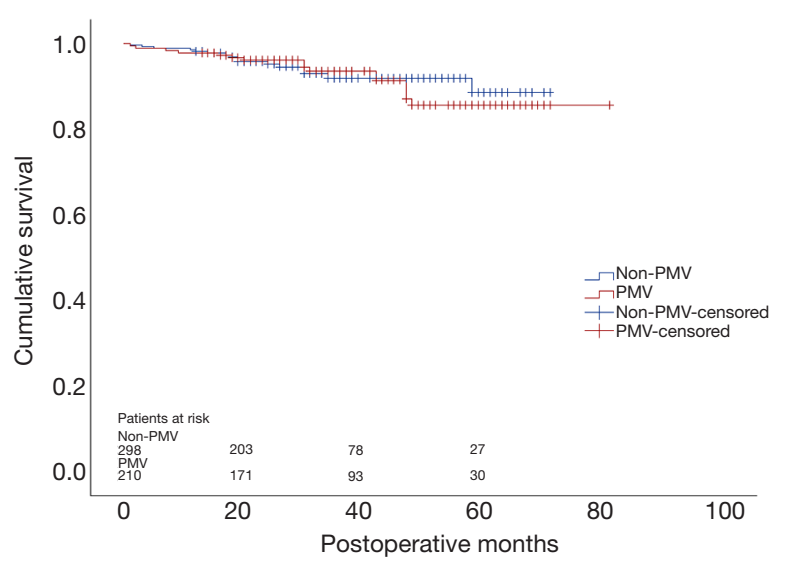

Figure 1 Kaplan-Meier estimation analysis in patients with and without PMV after surgery for DeBakey type I aortic dissection (Log rank $\mathrm{P}=0.728$ ). PMV, prolonged mechanical ventilation.

proinflammatory mediators. A lower platelet count may indicate an exhaustion after response to extensive falselumen thrombosis during the process of acute aortic dissection and systemic inflammation.

This study was the first to identify an elevated level of preoperative $\mathrm{sCyC}$ level as a risk factor for $\mathrm{PMV}$. This discovery suggested that preoperative renal dysfunction could affect postoperative intubation duration. Several studies have examined the association between preoperative renal function and PMV. Reddy and colleagues (2) showed that preoperative $\mathrm{sCr}$ was an independent risk factor for PMV after cardiac surgery. Similarly, Lei et al. (14) also suggested that preoperative $\mathrm{sCr}$ was a strong predictor for prolonged ventilation after aortic arch surgery. However, previous reports showed that $\mathrm{s} C y C$ was a better indicator of kidney function than $\mathrm{sCr}$ and predicted acute kidney injury 1-2 days earlier than the conventional clinical diagnosis $(15,16)$. The results of our study demonstrated that preoperative $\mathrm{sCyC}$ levels rather than $\mathrm{sCr}$ levels were an independent risk factor for $\mathrm{PMV}$ after aortic surgery.

Prolonged CPB time was identified as another predictor for PMV. Lei and colleagues (14) found that prolonged $\mathrm{CPB}$ time was a strong predictor for PMV after aortic arch surgery with DHCA. Other studies have suggested that patients avoiding the use of $\mathrm{CPB}$ when undergoing coronary artery surgery benefited from shorter ventilation times $(17,18)$. Multiple factors including systemic inflammatory response, lung interstitial edema, cytotoxic mediators, emboli and reperfusion injury can precipitate pulmonary dysfunction after CPB (19). Our data suggested that shorting the $\mathrm{CPB}$ duration as much as possible might improve patients' prognosis after DeBakey type I aortic dissection repair surgery.

The incidence of PMV in literatures varies from 24 hours to 14 days depends on the definition (20-22). The enrolled patients who underwent DeBakey type I aortic dissection surgery were characterized as PMV in our study based on the cut-off value of $48 \mathrm{~h}$. In addition, this study demonstrated that patients with PMV suffered from reduced early survival rate, longer ICU and hospital stay. These findings were consistent with previous studies $(4,23)$, which showed a higher in-hospital mortality rate for patients with PMV. For long-term outcomes, there was no significant difference identified between patients with or without PMV, which might be the short follow-up period after the surgery. The associations we discovered in this study helped us to better understand the risk factors for and outcomes of PMV after DeBakey type I aortic dissection repair surgery and shed light upon optimizing the perioperative management strategies.

\section{Limitations}

This study had several limitations. Firstly, the nature of the study was a retrospective analysis of prospectively collected data and might presented with some inherent biases. Secondly, data of important preoperative inflammatory cytokines (i.e., interleukin-6, interleukin-8, and TNF- $\alpha$ ) was lacking. Thirdly, some severe postoperative complications that might associate with PMV were not included in the multivariable logistic analysis model.

\section{Conclusions}

Our data suggested that PMV after DeBakey type I aortic dissection repair surgery was a common complication and associated with increased short-term mortality rate but did not affect long-term mortality rate. Multiple regression model suggested that elevated preoperative leukocyte count, increased preoperative serum cystatin $\mathrm{C}$ level, lower preoperative platelet count and longer $\mathrm{CPB}$ duration were potential risk factors for PMV. This study not only helped us to optimize perioperative management strategies, but also provided insights into understanding the pathogenesis of aortic dissection repair surgery associated PMV.

\section{Acknowledgments}

Funding: None. 


\section{Footnote}

Reporting Checklist: The authors have completed the STROBE reporting checklist. Available at http://dx.doi. org/10.21037/jtd-20-2736

Data Sharing Statement: Available at http://dx.doi. org/10.21037/jtd-20-2736

Conflicts of Interest: All authors have completed the ICMJE uniform disclosure form (available at http://dx.doi. org/10.21037/jtd-20-2736). The authors have no conflicts of interest to declare.

Ethical Statement: The authors are accountable for all aspects of the work in ensuring that questions related to the accuracy or integrity of any part of the work are appropriately investigated and resolved. The ethics committee of Nanjing Drum Tower hospital approved this retrospective study (No. BL2014004) and waived the need for individual informed consent due to the nature of this study. The study was conducted in accordance with the Declaration of Helsinki (as revised in 2013).

Open Access Statement: This is an Open Access article distributed in accordance with the Creative Commons Attribution-NonCommercial-NoDerivs 4.0 International License (CC BY-NC-ND 4.0), which permits the noncommercial replication and distribution of the article with the strict proviso that no changes or edits are made and the original work is properly cited (including links to both the formal publication through the relevant DOI and the license). See: https://creativecommons.org/licenses/by-nc-nd/4.0/.

\section{References}

1. Hasegawa $Y$, Ishikawa S, Ohtaki A, et al. Impaired lung oxygenation in acute aortic dissection. J Cardiovasc Surg (Torino) 1999;40:191-5.

2. Reddy SL, Grayson AD, Griffiths EM, et al. Logistic risk model for prolonged ventilation after adult cardiac surgery. Ann Thorac Surg 2007;84:528-36.

3. Li CN, Chen L, Ge YP, et al. Risk factors for prolonged mechanical ventilation after total aortic arch replacement for acute DeBakey type I aortic dissection. Heart Lung Circ 2014;23:869-74.

4. Jin M, Ma WG, Liu S, et al. Predictors of Prolonged Mechanical Ventilation in Adults After Acute Type-A
Aortic Dissection Repair. J Cardiothorac Vasc Anesth 2017;31:1580-7.

5. Cislaghi F, Condemi AM, Corona A. Predictors of prolonged mechanical ventilation in a cohort of 5123 cardiac surgical patients. Eur J Anaesthesiol 2009;26:396-403.

6. Wang Y, Xue S, Zhu H. Risk factors for postoperative hypoxemia in patients undergoing Stanford A aortic dissection surgery. J Cardiothorac Surg 2013;8:118.

7. Xue Y, Pan J, Cao H, et al. Different aortic arch surgery methods for type A aortic dissection: clinical outcomes and follow-up results. Interact Cardiovasc Thorac Surg 2020;31:254-62.

8. Wang $Z$, Ge $M$, Chen $T$, et al. Independent risk factors and the long-term outcomes for postoperative continuous renal replacement treatment in patients who underwent emergency surgery for type A acute aortic dissection. J Cardiothorac Surg 2020;15:100.

9. Doerschuk CM. Mechanisms of leukocyte sequestration in inflamed lungs. Microcirculation 2001;8:71-88.

10. Pararajasingam R, Nicholson ML, Bell PR, et al. Noncardiogenic pulmonary oedema in vascular surgery. Eur J Vasc Endovasc Surg 1999;17:93-105.

11. Xu CE, Zou CW, Zhang MY, et al. Effects of high-dose ulinastatin on inflammatory response and pulmonary function in patients with type-A aortic dissection after cardiopulmonary bypass under deep hypothermic circulatory arrest. J Cardiothorac Vasc Anesth 2013;27:479-84.

12. Alexiou C, Tang AA, Sheppard SV, et al. The effect of leucodepletion on leucocyte activation, pulmonary inflammation and respiratory index in surgery for coronary revascularisation: a prospective randomised study. Eur J Cardiothorac Surg 2004;26:294-300.

13. Zarbock A, Ley K. The role of platelets in acute lung injury (ALI). Front Biosci (Landmark Ed) 2009;14:150-8.

14. Lei Q, Chen L, Zhang Y, et al. Predictors of prolonged mechanical ventilation after aortic arch surgery with deep hypothermic circulatory arrest plus antegrade selective cerebral perfusion. J Cardiothorac Vasc Anesth 2009;23:495-500.

15. Dharnidharka VR, Kwon C, Stevens G. Serum cystatin $\mathrm{C}$ is superior to serum creatinine as a marker of kidney function: a meta-analysis. Am J Kidney Dis 2002;40:221-6.

16. Yim H, Kym D, Seo DK, et al. Serum cystatin C and microalbuminuria in burn patients with acute kidney injury. Eur J Clin Invest 2015;45:594-600. 
17. Güler M, Kirali K, Toker ME, et al. Different CABG methods in patients with chronic obstructive pulmonary disease. Ann Thorac Surg 2001;71:152-7.

18. Staton GW, Williams WH, Mahoney EM, et al. Pulmonary outcomes of off-pump vs on-pump coronary artery bypass surgery in a randomized trial. Chest 2005;127:892-901.

19. Gasparovic H, Plestina S, Sutlic Z, et al. Pulmonary lactate release following cardiopulmonary bypass. Eur J Cardiothorac Surg 2007;32:882-7.

20. Légaré JF, Hirsch GM, Buth KJ, et al. Preoperative prediction of prolonged mechanical ventilation following coronary artery bypass grafting. Eur J Cardiothorac Surg
2001;20:930-6.

21. Pappalardo F, Franco A, Landoni G, et al. Long-term outcome and quality of life of patients requiring prolonged mechanical ventilation after cardiac surgery. Eur J Cardiothorac Surg 2004;25:548-52.

22. Kollef MH, Wragge T, Pasque C. Determinants of mortality and multiorgan dysfunction in cardiac surgery patients requiring prolonged mechanical ventilation. Chest 1995;107:1395-401.

23. Schechter MA, Shah AA, Englum BR, et al. Prolonged postoperative respiratory support after proximal thoracic aortic surgery: Is deep hypothermic circulatory arrest a risk factor? J Crit Care 2016;31:125-9.
Cite this article as: Ge M, Wang Z, Chen T, Cheng Y, Ye J, Lu L, Chen C, Wang D. Risk factors for and outcomes of prolonged mechanical ventilation in patients received DeBakey type I aortic dissection repairment. J Thorac Dis 2021;13(2):735742. doi: $10.21037 /$ jtd-20-2736 